\title{
High frame rate synthetic aperture vector flow imaging for transthoracic
} echocardiography.

Villagómez Hoyos, Carlos Armando; Stuart, Matthias Bo; Bechsgaard, Thor ; Nielsen, Michael Bachmann; Jensen, Jørgen Arendt

\section{Published in:}

Proceedings of SPIE

Link to article, DOI:

$10.1117 / 12.2216707$

Publication date:

2016

Document Version

Peer reviewed version

Link back to DTU Orbit

Citation (APA):

Villagómez Hoyos, C. A., Stuart, M. B., Bechsgaard, T., Nielsen, M. B., \& Jensen, J. A. (2016). High frame rate synthetic aperture vector flow imaging for transthoracic echocardiography. In N. Duric, \& B. Heyde (Eds.), Proceedings of SPIE (Vol. 9790). [979004] SPIE - International Society for Optical Engineering. https://doi.org/10.1117/12.2216707

\section{General rights}

Copyright and moral rights for the publications made accessible in the public portal are retained by the authors and/or other copyright owners and it is a condition of accessing publications that users recognise and abide by the legal requirements associated with these rights.

- Users may download and print one copy of any publication from the public portal for the purpose of private study or research.

- You may not further distribute the material or use it for any profit-making activity or commercial gain

- You may freely distribute the URL identifying the publication in the public portal 


\title{
High frame rate synthetic aperture vector flow imaging for transthoracic echocardiography
}

\author{
Carlos A. Villagómez-Hoyos ${ }^{a}$, Matthias B. Stuart ${ }^{a}$, Thor Bechsgaard ${ }^{b}$ \\ Michael Bachmann Nielsen ${ }^{b}$ and Jørgen Arendt Jensen ${ }^{a}$ \\ ${ }^{a}$ Center for Fast Ultrasound Imaging, Department of Electrical Engineering, \\ Technical University of Denmark, DK-2800 Lyngby, Denmark; \\ ${ }^{b}$ Department of Radiology, Rigshospitalet, Copenhagen University Hospital, DK-2100 \\ Copenhagen, Denmark;
}

\begin{abstract}
This work presents the first in vivo results of 2-D high frame rate vector velocity imaging for transthoracic cardiac imaging. Measurements are made on a healthy volunteer using the SARUS experimental ultrasound scanner connected to an intercostal phased-array probe. Two parasternal long-axis view (PLAX) are obtained, one centred at the aortic valve and another centred at the left ventricle. The acquisition sequence was composed of 3 diverging waves for high frame rate synthetic aperture flow imaging. For verification a phantom measurement is performed on a transverse straight $5 \mathrm{~mm}$ diameter vessel at a depth of $100 \mathrm{~mm}$ in a tissue-mimicking phantom. A flow pump produced a $2 \mathrm{ml} / \mathrm{s}$ constant flow with a peak velocity of $0.2 \mathrm{~m} / \mathrm{s}$. The average estimated flow angle in the ROI was $86.22^{\circ} \pm 6.66^{\circ}$ with a true flow angle of $90^{\circ}$. A relative velocity bias of $-39 \%$ with a standard deviation of $13 \%$ was found. In-vivo acquisitions show complex flow patterns in the heart. In the aortic valve view, blood is seen exiting the left ventricle cavity through the aortic valve into the aorta during the systolic phase of the cardiac cycle. In the left ventricle view, blood flow is seen entering the left ventricle cavity through the mitral valve and splitting in two ways when approximating the left ventricle wall. The work presents 2-D velocity estimates on the heart from a non-invasive transthoracic scan. The ability of the method detecting flow regardless of the beam angle could potentially reveal a more complete view of the flow patterns presented on the heart.
\end{abstract}

Keywords: Medical Ultrasound, Vector Flow Imaging, Cardiac Imaging, Blood Flow, Synthetic Aperture

\section{INTRODUCTION}

Doppler echocardiography is an important tool for noninvasive assessment of cardiac function and management of cardiac patients. ${ }^{1}$ It provides valuable insights to the pathophysiology of cardiac diseases and contributes to clinical decision making. Blood flow, pressures, and pressure gradients can be derived to assess systolic and diastolic performance as well as valve function. Therefore, accurate assessment of cardiac function during all phases of the heart cycle is a central issue in cardiac imaging.

Conventional cardiac ultrasound technologies allow for the measurement of blood or tissue velocity along the ultrasound beam at frame rates ranging from 20 to $100 \mathrm{~Hz}$. However, a tradeoff between the field of view and the achievable frame rate is present due to the line per line nature of these conventional systems. ${ }^{2}$

High frame rate techniques, such as synthetic aperture and plane wave imaging, present an alternative to conventional techniques. ${ }^{3-5}$ These techniques, which make use of wider transmit beams, allow for breaking the usual tradeoff between field of view and temporal resolution. ${ }^{6}$ In transthoracic cardiac imaging, the use of high temporal resolutions for B-mode and 1-D Doppler modalities have been recently introduced..$^{7,8}$

High frame rate 2-D vector velocity algorithms provides a more complete representation of the blood flow, but have only been implemented on linear arrays. ${ }^{9-12}$ Studies of the heart, therefore, have been limited to intraoperative ${ }^{13}$ or newborn ${ }^{14}$ scans with the heart on a shallower depth. In transthoracic cardiac imaging, the

Further author information: Send correspondence to Villagomez-Hoyos, E-mail: cavh@elektro.dtu.dk. 
heart is located at deeper depths and available only through limited acoustic windows through the rib cage. Therefore, the use of a smaller phased array is preferred. Diverging waves based algorithms, such as synthetic aperture, presents an attractive solution to high frame rate transthoracic scanning. Diverging waves present wider field of view than their focused counterpart and are, therefore, more suitable to smaller apertures such as the one present on the phased array.

Recently, the introduction of a novel 2-D high frame rate vector velocity algorithm, based on synthetic aperture,${ }^{9}$ has been investigated for linear arrays. ${ }^{15}$ The algorithm has shown accurate estimates with hundreds to thousands of frames per second over large field of views. This accurate estimation of 2-D velocity vectors provides the removal of the need for manual angle correction, which would be difficult or impossible in conventional systems due to the complex nature of the hemodynamics in the heart.

In this paper, the first known demonstration of in vivo high frame rate cardiac vector flow imaging (VFI) using a noninvasive, transthoracic approach is presented. A brief theoretical description of the vector velocity estimation and a energy based echo-canceler is presented in Section 2. Section 3 describes the methods and imaging setup used in the pre-clinical testing. In Section 4, the flow phantom and in vivo results are presented and discussed, and Section 5 discusses the conclusions of this work.

\section{THEORY}

\subsection{Velocity estimation}

The velocity estimation idea, illustrated in Fig. 1, is based on the directional beamforming approach proposed by Jensen et al. ${ }^{16}$ The velocity of a moving scatterer, represented by an elliptical point spread function (middle), is estimated by measuring the scatterer displacement during a sequence of consecutive emissions. The emission sequence is composed of at least two distinct transmit/receive aperture pairs, illustrated as the distinctly rotated point spread functions (PSF). For each emission, the received multichannel data are focused in a directional line $g\left(r, \theta_{m}\right)$, represented in Fig. 1 (bottom) as the semi-elliptic sections on which the directional line cuts the elliptic PSFs. The directional velocity estimation is then performed by correlating the directional lines $g\left(r, \theta_{m}\right)$ from the same type of rotated PSFs and obtaining a displacement distance $d$. Dividing this displacement by the time between emissions $T_{\text {eff }}$ gives a velocity magnitude.

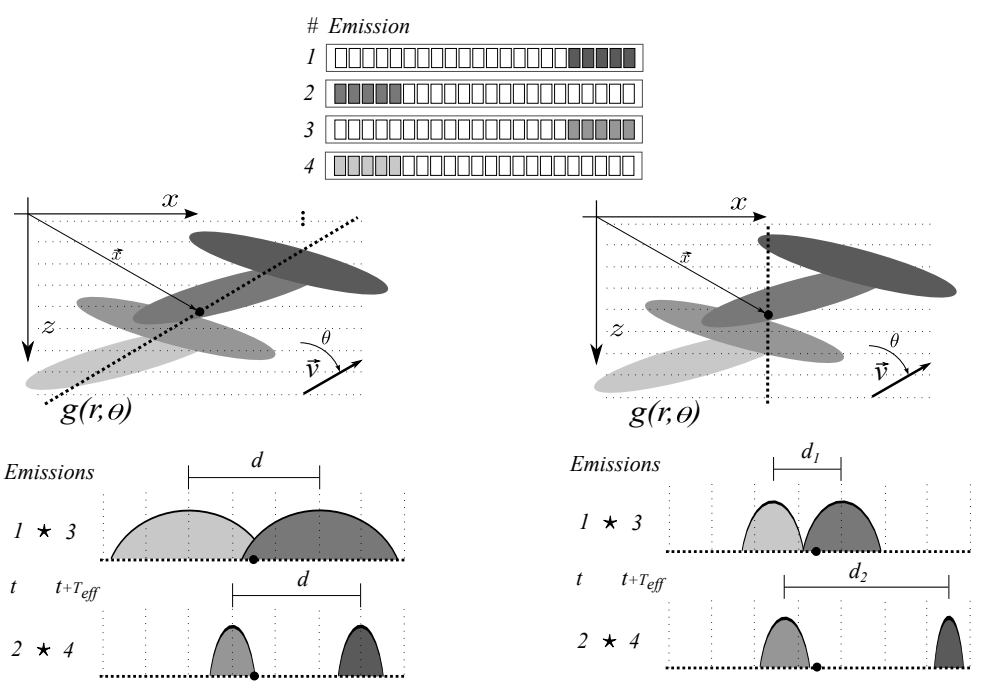

Figure 1: Point spread functions of a moving scatterer represented by ellipses. A directional line $g\left(r, \theta_{m}\right)$ is beamformed in the direction of the movement (left) and in an incorrect direction (right). Semi-elliptic sections on which the directional line cuts the rotated elliptic PSFs is shown at the bottom.

The measured velocity magnitude is only correct, if the directional line coincides with the direction of the movement as seen in Fig 1(left). In any other case, the obtained velocity magnitude will be erroneous, with an 
error dependent on the beam-to-flow-angle as seen in Fig. 1 (right). Therefore, for estimating the true velocity direction and magnitude, a set of angular velocity functions are calculated from a polar grid $g\left(r, \theta_{m}\right)$, as shown in the top of Fig. 2. The angular velocity function is the estimated velocities in each beamformed direction, where consecutive beamformed lines are cross-correlated and the displacement is detected. By dividing with the time between emitted signals, the magnitude of the angle velocity function at that beamformed angle is obtained. To obtain the true flow direction from the calculated angle velocity functions $\tilde{V}\left(\vec{x}, t, \theta_{m}\right)$, a set of $M$ distinct steered PSF is needed. The true flow direction is estimated as the angle at the intersection of the angular velocity functions, see Fig. 2. Only two distinct steered PSFs are needed for a 2-D velocity estimation. However, in practice the use of a higher number of transmit/receive aperture combinations increases the robustness of the estimator.

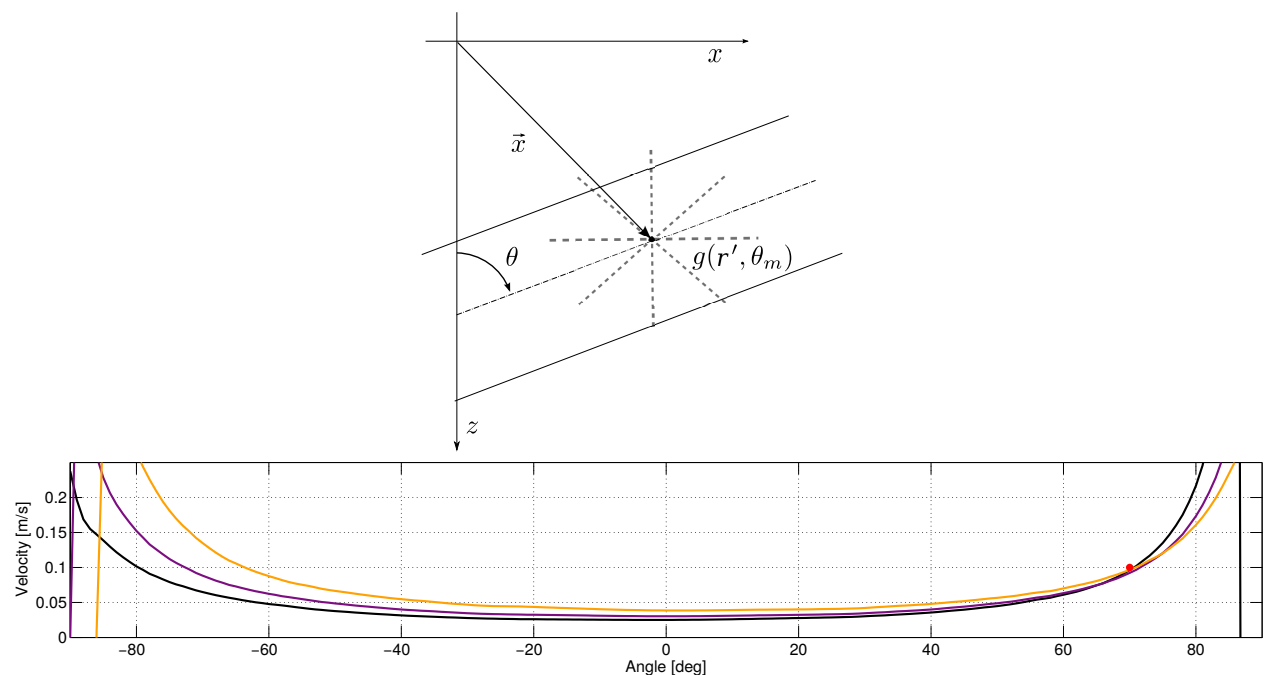

Figure 2: Directional beamformed lines in a polar grid (top). The angle velocity functions intersecting at the true flow direction shown as a red dot (bottom).

To calculate the intersection a two step approach is employed; first the closest coarse angles, where the intersection takes place, are selected. Then, the angle is refined by curve fitting between these two points and analytically finding the intersection angle.

In the first step, the closest coarse angles are selected by the use of a minimum distance criterion (MDC). The distance to be minimized in the MDC is defined as the sum of the normalized difference between the angle velocity functions:

$$
\theta_{M D C_{1}}=\underset{\theta_{m}}{\operatorname{Arg} \min } \sum_{k=1}^{M-1} \sum_{l=k+1}^{M}\left|\frac{V_{k}\left(\theta_{m}\right)-V_{l}\left(\theta_{m}\right)}{\min \left(V_{k}\left(\theta_{m}\right), V_{l}\left(\theta_{m}\right)\right)}\right|
$$

In the second step, the selected coarse angle $\theta_{M D C_{1}}$ and the adjacent coarse angle with the second lowest MDC value $\theta_{M D C_{2}}$ are used to extract the segments of the curves used to refine the angle estimate. The points $\theta_{M D C_{1}}$ and $\theta_{M D C_{2}}$ are used for the an inverse cosine curve fitting. The intersection of each pair of angle velocity functions is then estimated, and the angle estimate is the median of these values

$$
\theta=\operatorname{median}\left(\theta_{(l, k)}\right) .
$$

where $l$ and $k$ are the different pairs of fitted $V_{M}$ curves.

After the angle has been obtained, the velocity magnitude is obtained by beamforming a directional line at the estimated angle. The final velocity magnitude is obtained using the summation of beamformed lines from distinct emissions instead of individual emissions. The summation generates a high resolution line as also known from synthetic aperture flow techniques. ${ }^{9}$ 


\subsection{Energy-based tissue echo-canceler}

A major challenge in blood velocity estimation is the cancellation of the tissue signal, thereby enhancing the low signal from blood cells. This is especially important in the heart, where the tissue velocities are significantly higher than in other organs. This problem is aggravated for vector velocity estimation as the frequency content of the signal from blood is strongly dependent on the flow angle, ${ }^{17}$ thus, it is in general difficult to choose a single cut-off frequency that discriminates between moving tissue and the flowing structure. Therefore, to avoid the selection of a cut-off frequency a energy-based filter is proposed.

The energy filter is used to attenuate tissue motion that otherwise overpowers the signal from flowing structures. The filter operates on the velocity spectrum components. However, instead of selecting a conventional frequency cut-off threshold it selects an energy cut off threshold as shown in Fig. 3. The energy cut-off threshold can be adaptively selected as suggested by Siggia et al, ${ }^{18}$ or it can be a pre-set value heuristically calculated given the characteristics of the imaging system.

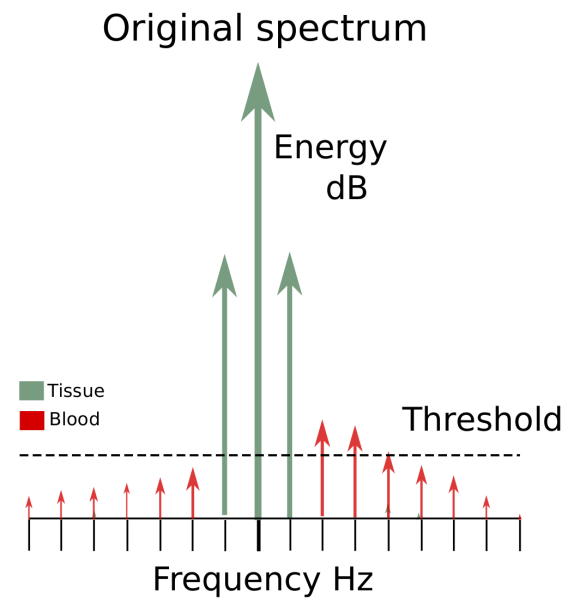

a)
Filtered spectrum

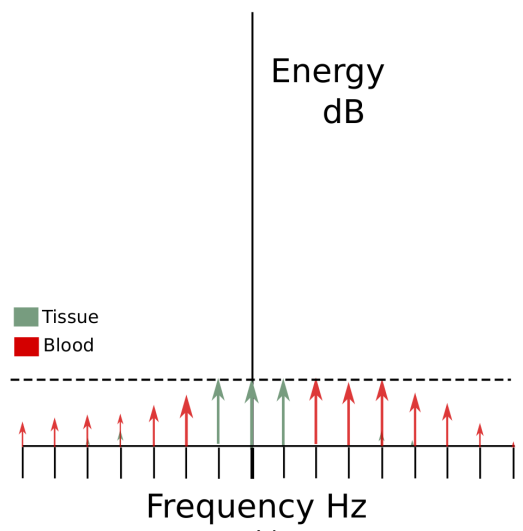

b)

Figure 3: Illustration of the energy based filter on the velocity spectrum (a). The tissue energy is represented by the green arrows, and the blood energy by the red arrows. An energy level threshold is used to limit the amount of energy from the tissue scatterers (b).

The energy filter operates on the basis that blood signal have lower energy and broader velocity spectrum compared to tissue signals. Therefore, by limiting the amplitude of the velocity spectrum the energy of the tissue signal is overpowered by the energy of a more broad-band blood signal. Thus, the selected limiting threshold is the mean amplitude level of the blood signal spectrum.

The implementation of the pre-set energy filter is described as follows:

1. Fourier transform the RF data to obtain velocity spectrum.

2. Energy limit the spectrum amplitude to the pre-determined threshold conserving the phase information of the signals.

3. Inverse Fourier transform to obtain the filtered RF data.

\section{METHOD}

A 128-element phased array probe is used for the investigation. A synthetic aperture sequence is used to acquire the flow data set. The flow sequence is implemented using the full 128-element aperture for each emission to emulate a spherical wave emanating from a virtual point source located behind the aperture. The transmitted wavefront is directed towards a region of interest (ROI), so the ROI is completely insonified in every emission 
Table 1: Transducer and Acquisition parameters

\begin{tabular}{llllll}
\hline \multicolumn{2}{c}{ Transducer } & & & \multicolumn{2}{c}{ Transmit Parameters } \\
\cline { 1 - 2 } \cline { 5 - 6 } Parameter & Value & & Parameter & Value \\
\hline Transducer type & Phased array & & Excitation signal & Neriod sine \\
Number of transducer elements & 128 & & & Number of emitting elements & 128 \\
Transducer element pitch & $0.22 \mathrm{~mm}$ & & Apodization window & Hanning \\
Transducer element kerf & $0.022 \mathrm{~mm}$ & & Number of distinct emissions & 3 \\
Transducer element height & $15 \mathrm{~mm}$ & & Pulse repetition frequency $(\mathrm{PRF})$ & $6.2 \mathrm{KHz}$ \\
Elevation focus & $85 \mathrm{~mm}$ & & F-number & -1.6 \\
Center frequency & $3.5 \mathrm{MHz}$ & & & \\
\hline
\end{tabular}

(Fig. 4). A 3-cycle sinusoidal pulse weighted by a $50 \%$ Tukey window is used as the excitation waveform. An effective pulse repetition time is $T_{e f f}=\frac{T_{p r f}}{3}$, where $T_{p r f}$ is the inverse of the system pulse repetition frequency (PRF). The transducer and acquisition parameters are listed in Table 1.
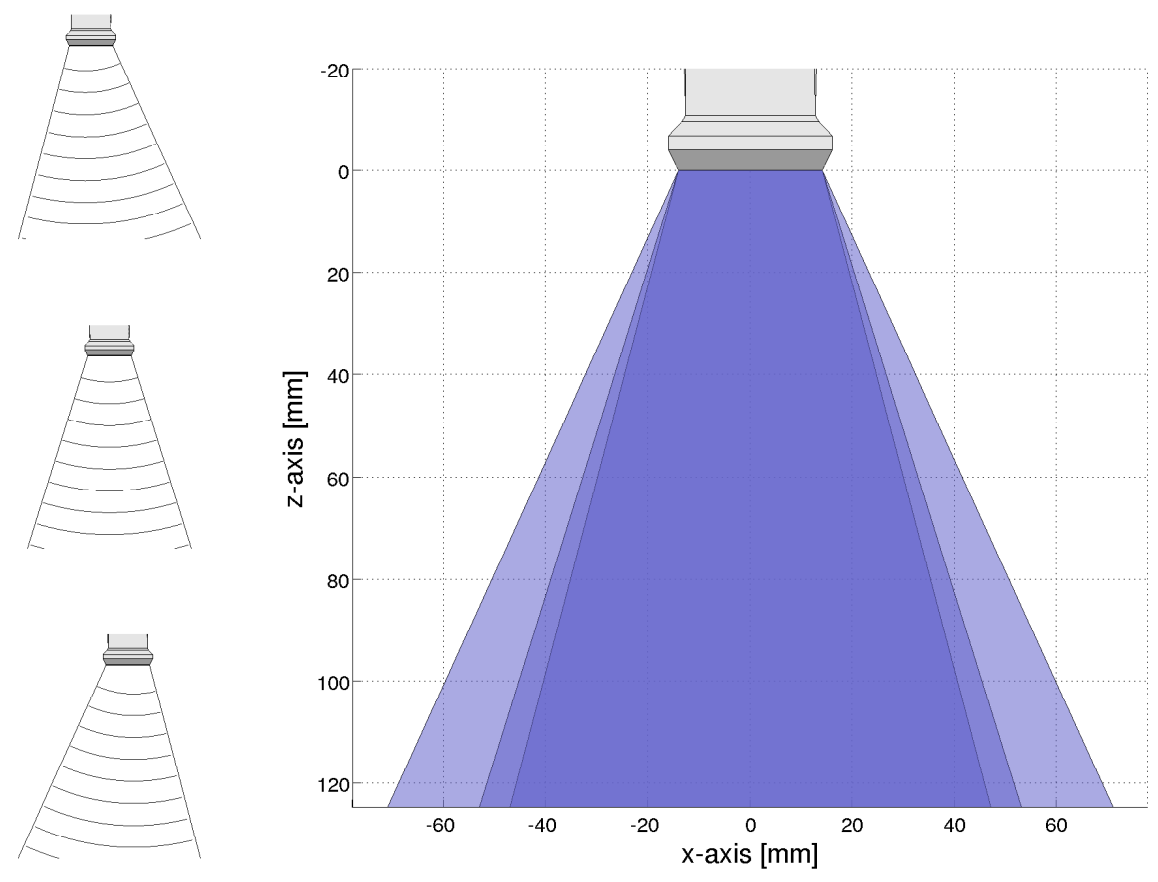

Figure 4: Example of spherical waves emanated from the first, second, and third emission used in the flow sequence (left). Overlay of the insonified area from each emission, and the actual region of interest shown in a darker shade (right).

The experimental ultrasound scanner SARUS ${ }^{19}$ is used for acquiring data. The system acquires RF data from the individual transducer channels, and data are transferred to a computing cluster, where they are stored and processed off-line. The received RF data contains 128 channels sampled at $35 \mathrm{MHz}$ with 12 bits resolution.

\subsection{Flow phantom}

Measurements are made in a straight vessel phantom. A Shelley Medical software controlled pump (Toronto, Canada) is used to circulate blood-mimicking fluid ${ }^{20}$ (Danish Phantom Design, Frederikssund, Denmark) in a 
closed loop circuit. The system is set for a constant flow rate of $2 \mathrm{ml} / \mathrm{s}$ in a $5 \mathrm{~mm}$ diameter wide vessel. The straight vessel is embedded in tissue-mimicking material and is located $100 \mathrm{~mm}$ in depth and is completely transverse to the ultrasound beam.

\subsection{In-vivo}

An in-vivo acquisition is performed after approval by The Danish National Committee on Biomedical Research Ethics. A healthy volunteer with no history of cardiovascular disease (29 year old man) is scanned after informed consent. An ultrasound scan sequence, as described in Section 3, is acquired on a parasternal long-axis view (PLAX). The scans are obtained with the patient in left lateral decubitus position and the transducer placed near the sternum in the left third or fourth intercostal space.

Intensity measurements are carried out prior to the scan, since measured intensities must satisfy limits regulated by the U.S. Food and Drug Administration (FDA). ${ }^{21}$ These limits concern the mechanical index, $M I \leq 1.9$, the derated spatial-peak-temporal-average intensity, $I_{\text {spta }} \leq 720 \mathrm{~mW} / \mathrm{cm}^{2}$. The measured values using the scheme described by Jensen, ${ }^{22}$ are $M I=0.62$ and a $I_{\text {spta }}=106.8 \mathrm{~mW} / \mathrm{cm}^{2}$, which are both below the FDA limits. The transducer surface temperature was also tested, where the transducer surface did not exceeded an increase of $30^{\circ}$ in air or $10^{\circ}$ Celsius in simulated usage test.

\section{RESULTS}

The generated 2-D velocity vectors are overlayed on the high frame rate B-mode image frames. The overlaying colors are change depending on the flow magnitude and direction of the estimated flow, and are in accordance to the color wheel map provided in the lower right corner of each frame. The estimates are generated at a frame rate of $2,000 \mathrm{~Hz}$.

\subsection{Phantom study}

Results from the flow phantom acquisition are shown in Fig. 5. The flow within the vessel is masked with a marked ROI. The angle of flow in the ROI had a mean angle of $86.22 \pm 6.66$ degrees at one standard deviation. The relatively small bias may be caused by misalignment in the physical setup. The measured velocities presented distinct biases depending on their spatial position. The worst case is at the middle of the vessel, where the bias reaches $-34 \%$ with standard deviations of $32 \%$. For the left and right profiles the relative bias are $-33 \%$ and $23 \%$ with a standard deviation of $-39 \%$ and $13 \%$, respectively. The source of this underestimation respect to the true reference is unknown and must be further investigated.

\subsection{In-vivo}

A parasternal long-axis (PLAX) view centred on the aortic valve (AoV) during systole is shown in Fig. 6 (top). A high quality B-mode PLAX view obtained using a commercial scanner seconds prior to the experimental scan is presented in the upper right corner as an anatomical reference. The left ventricle (LV) is observed on the left of image sector, while the AoV and aorta are observed on the right. The blood flow in Fig. 6 (top) is detected flowing from the LV cavity through the AoV into the aorta artery. The flow direction is consistent with the anatomy as the flow is detected parallel to the artery wall and heading towards the aorta during the systole at velocity magnitudes of around $1 \mathrm{~m} / \mathrm{s}$.

A second view, in Fig. 6 (bottom), shows a centred LV during the filling state with the mitral valve located in the right left corner, more clearly seen in the reference image. Blood flow is seen emanating from the mitral valve and splitting in two ways when approximating the left ventricle wall. A complex flow pattern is formed when the blood flow fills the left ventricle showing a consistent behaviour with the mitral valve position. 


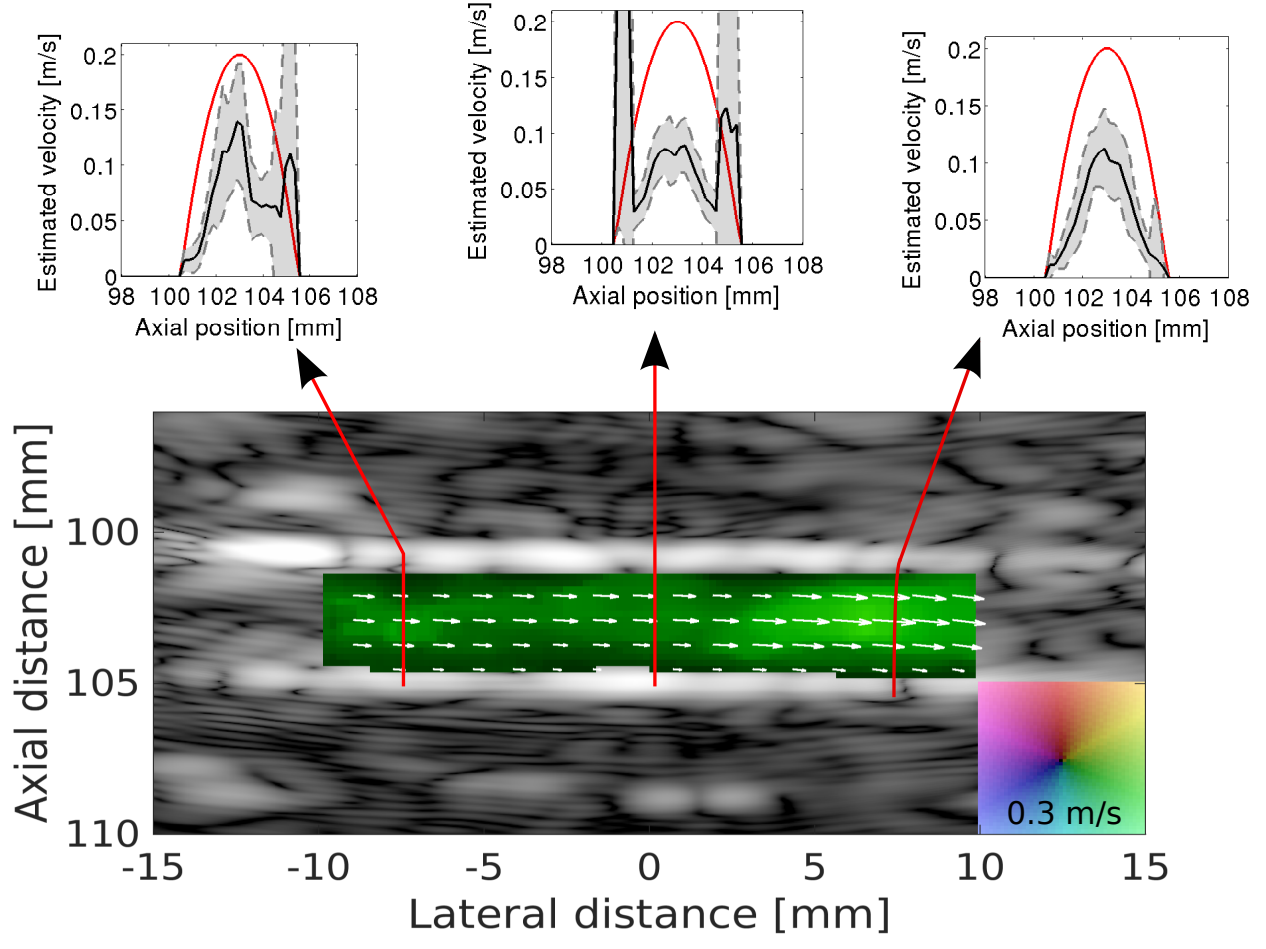

Figure 5: Mean velocity profiles and standard deviations for three different positions (top). 2-D velocity vectors in a vessel phantom at $100 \mathrm{~mm}$ depth, with arrows indicating velocity direction and relative magnitude with corresponding 2-D color wheel map (bottom).

\section{CONCLUSION AND PERSPECTIVES}

In this work, we investigated the ability of synthetic aperture flow techniques to map 2-D velocity vectors of ventricular blood flow with a frame rate of 2,000 images per second over a full two-dimensional sector view. A major advantage of the technique is the ability to estimate flow regardless of the beam-to-flow angle. The technique also provides velocity information at every location in the image simultaneously and at high frame rate.

Finally, vector velocity techniques have the capability to improve guidance and feedback to researchers and cardiologists through improved visualization of turbulence and the removal of the angle-correction of conventional spectral Doppler estimation.

\section{REFERENCES}

1. R. A. Nishimura and A. J. Tajik, "Evaluation of diastolic filling of left ventricle in health and disease: Doppler echocardiography is the clinician rosetta stone," J. Am. Coll. Cardiol. 30(1), pp. 8-18, 1997.

2. N. S. Anavekar and J. K. Oh, "Doppler echocardiography: A contemporary review," J. Cardiology 54, pp. 347-358, 2009.

3. J. T. Ylitalo, "A fast ultrasonic synthetic aperture imaging method: application to NDT," Ultrasonics , pp. 331-333, 1996.

4. J. Y. Lu, "2D and 3D high frame rate imaging with limited diffraction beams," IEEE Trans. Ultrason., Ferroelec., Freq. Contr. 44, pp. 839-855, 1997.

5. L. Sandrin, S. Catheline, M. Tanter, X. Hennequin, and M. Fink, "Time-resolved pulsed elastography with ultrafast ultrasonic imaging," Ultrason. Imaging 21(4), pp. 259-272, 1999.

6. S. I. Nikolov and J. A. Jensen, "In-vivo Synthetic Aperture Flow Imaging in Medical Ultrasound," IEEE Trans. Ultrason., Ferroelec., Freq. Contr. 50(7), pp. 848-856, 2003.

7. M. B. Stuart, J. Jensen, A. H. Brandt, S. I. Nikolov, M. B. Nielsen, and J. A. Jensen, "In-vivo synthetic aperture and plane wave high frame rate cardiac imaging," in Proc. IEEE Ultrason. Symp., pp. 1209-1212, 2014. 
8. B.-F. Osmanski, D. Maresca, E. Messas, M. Tanter, and M. Pernot, "Transthoracic ultrafast Doppler imaging of human left ventricular hemodynamic function," IEEE Trans. Ultrason., Ferroelec., Freq. Contr. 61, pp. 1268-1275, August 2014.

9. J. A. Jensen and S. I. Nikolov, "Directional synthetic aperture flow imaging," IEEE Trans. Ultrason., Ferroelec., Freq. Contr. 51, pp. 1107-1118, 2004.

10. J. Udesen, F. Gran, K. L. Hansen, J. A. Jensen, C. Thomsen, and M. B. Nielsen, "High frame-rate blood vector velocity imaging using plane waves: simulations and preliminary experiments," IEEE Trans. Ultrason., Ferroelec., Freq. Contr. 55(8), pp. 1729-1743, 2008.

11. J. Bercoff, G. Montaldo, T. Loupas, D. Savery, F. Meziere, M. Fink, and M. Tanter, "Ultrafast compound Doppler imaging: providing full blood flow characterization," IEEE Trans. Ultrason., Ferroelec., Freq. Contr. 58, pp. 134-147, January 2011.

12. B. Denarie, T. A. Tangen, I. K. Ekroll, N. Rolim, H. H. Torp, T. Bjastad, and L. Løvstakken, "Coherent plane wave compounding for very high frame rate ultrasonography of rapidly moving targets," IEEE Trans. Ultrason., Ferroelec., Freq. Contr. 32(7), pp. 1265-1276, 2013.

13. K. L. Hansen, H. Møller-Sørensen, M. M. Pedersen, P. M. Hansen, J. Kjaergaard, J. T. Lund, J. C. Nilsson, J. A. Jensen, and M. B. Nielsen, "First report on intraoperative vector flow imaging of the heart among patients with healthy and diseased aortic valves," Ultrasonics 56, pp. 243-250, 2014.

14. S. Fadnes, S. A. Nyrnes, H. Torp, and L. Lovstakken, "Shunt flow evaluation in congenital heart disease based on two-dimensional speckle tracking," Ultrasound Med. Biol. 40(10), pp. 2379-2391, 2014.

15. C. A. Villagomez-Hoyos, M. B. Stuart, K. L. Hansen, M. B. Nielsen, and J. A. Jensen, "Accurate angle estimator for high frame rate 2-D vector flow imaging," IEEE Trans. Ultrason., Ferroelec., Freq. Contr., p. Submitted, 2016.

16. J. A. Jensen, "Directional velocity estimation using focusing along the flow direction: I: Theory and simulation," IEEE Trans. Ultrason., Ferroelec., Freq. Contr. 50, pp. 857-872, 2003.

17. M. E. Anderson, "Vector flow estimator isomorphism and wall filter requirements," in Proc. SPIE Med. Imag., 4325, pp. 215-226, 2001.

18. A. D. Siggia and R. E. Passarelli, "Gaussian model adaptive processing (GMAP) for improved ground clutter cancellation and moment calculation," ERAD , pp. 67-73, 2004.

19. J. A. Jensen, O. Holm, L. J. Jensen, H. Bendsen, S. I. Nikolov, B. G. Tomov, P. Munk, M. Hansen, K. Salomonsen, J. Hansen, K. Gormsen, H. M. Pedersen, and K. L. Gammelmark, "Ultrasound research scanner for real-time synthetic aperture image acquisition," IEEE Trans. Ultrason., Ferroelec., Freq. Contr. 52 (5), pp. 881-891, May 2005.

20. K. V. Ramnarine, D. K. Nassiri, P. R. Hoskins, and J. Lubbers, "Validation of a new blood mimicking fluid for use in Doppler flow test objects," Ultrasound Med. Biol. 24, pp. 451-459, 1998.

21. FDA, "Information for manufacturers seeking marketing clearance of diagnostic ultrasound systems and transducers," tech. rep., Center for Devices and Radiological Health, United States Food and Drug Administration, 2008.

22. J. A. Jensen, M. F. Rasmussen, M. J. Pihl, S. Holbek, C. A. Villagomez-Hoyos, D. P. Bradway, M. B. Stuart, and B. G. Tomov, "Safety assessment of advanced imaging sequences, I: Measurements," IEEE Trans. Ultrason., Ferroelec., Freq. Contr. 63(1), pp. 110-119, 2016. 

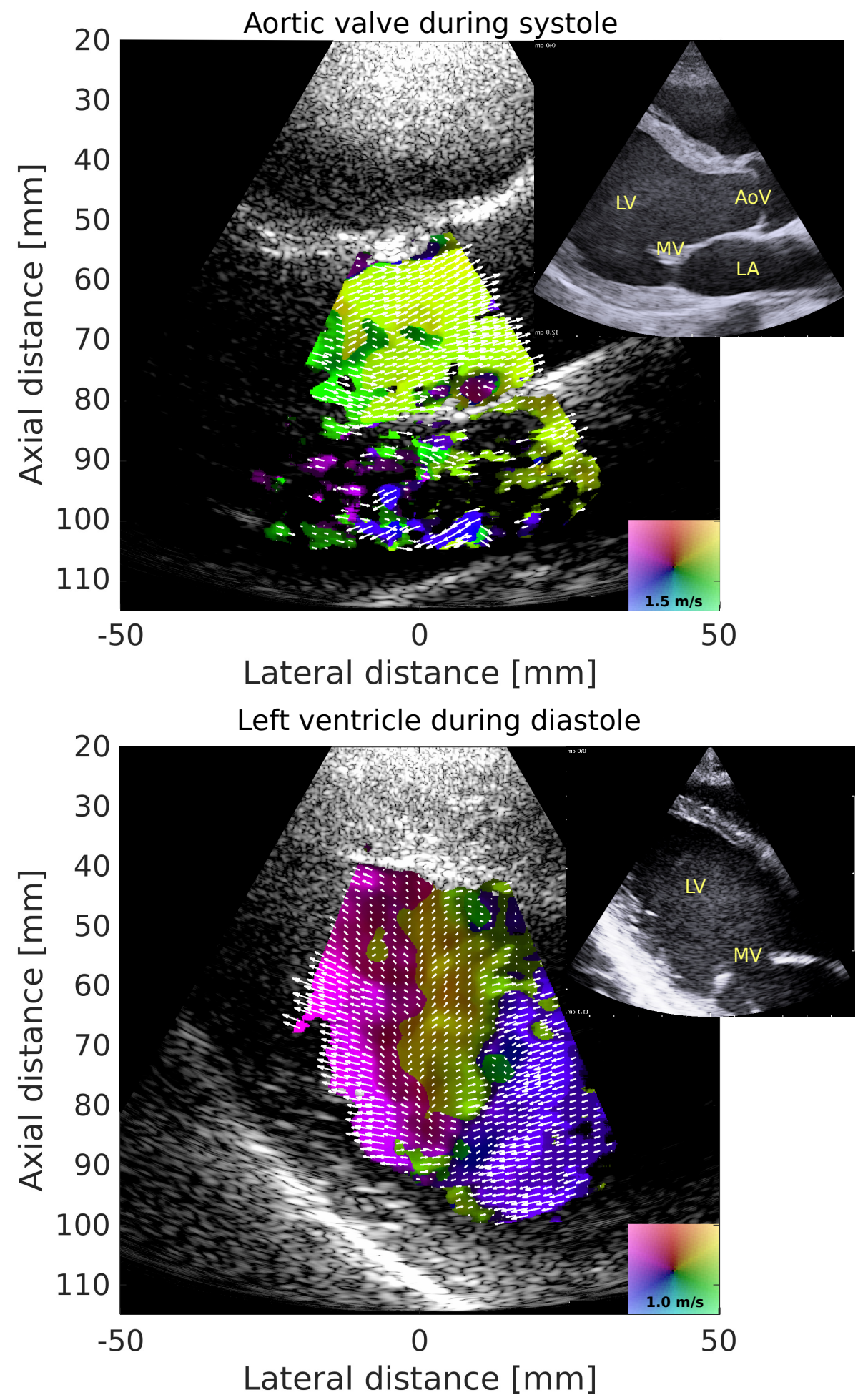

Figure 6: Vector Flow imaging frames of transthoracic scans on the heart. Two parasternal long-axis views of the heart; one centred on the aortic valve during systole (top) and the other on the left ventricle during the filling stage (bottom). A high quality B-mode on the right corner is provided as anatomical reference. 